\title{
Norois
}

Environnement, aménagement, société

$232 \mid 2014$

Modes de vie, modes d'habiter des aînés entre inclusion et exclusion

\section{Les grands ensembles résidentiels adaptés québécois destinés aux aînés. Une exploration de la marchabilité du quartier à l'aide d'un audit urbain}

The large residential habitats for seniors in Québec (Canada): An exploration of neighborhood walkability using an urban audit

\section{Sébastien Lord et Paula Negron-Poblete}

\section{(2) OpenEdition}

Journals

Édition électronique

URL : https://journals.openedition.org/norois/5157

DOI : 10.4000/norois. 5157

ISSN : 1760-8546

Éditeur

Presses universitaires de Rennes

Édition imprimée

Date de publication : 30 octobre 2014

Pagination : 35-52

ISBN : 978-2-7535-3974-7

ISSN : 0029-182X

Référence électronique

Sébastien Lord et Paula Negron-Poblete, «Les grands ensembles résidentiels adaptés québécois destinés aux aînés. Une exploration de la marchabilité du quartier à l'aide d'un audit urbain », Norois [En ligne], 232 | 2014, mis en ligne le 30 octobre 2016, consulté le 13 janvier 2022. URL : http:// journals.openedition.org/norois/5157 ; DOI : https://doi.org/10.4000/norois.5157 


Presses
Universitaires
de Rennes
www.pur-editions.fr
Revue en ligne : http://norois.revues.org

\title{
Les grands ensembles résidentiels adaptés québécois destinés aux aînés Une exploration de la marchabilité du quartier à l'aide d'un audit urbain
}

\author{
The Large Residential Habitats for Seniors in Québec (Canada): \\ An Exploration of Neighborhood Walkability Using an Urban Audit
}

\author{
Sébastien LorD*, Paula Negron-Poblete
}

*(auteur correspondant), Téléphone : (+001) 514.343.7215 Télécopieur : (+001) 514.343.2338, bureau 3015
Professeurs adjoints, Institut d'urbanisme, Faculté de l'aménagement, Université de Montréal - 2940, chemin de la Côte-
Sainte-Catherine, Montréal (Québec) H3T 1B9, Canada (sebastien.lord@umontreal.ca) (p.negron-poblete@umontreal.ca)

Résumé : Le vieillissement généralisé est évocateur des changements sociaux à l'échelle de plusieurs métropoles nord-américaines, où il est particulièrement visible dans les premières couronnes de banlieues pavillonnaires. Il s'agit ici d'une occasion unique de repenser la qualité urbaine de ces milieux de vie, où l'on dépend de la voiture pour se déplacer, et ce, dans une perspective de mobilité plus active. Une analyse exploratoire de l'environnement urbain immédiat de trois résidences pour personnes âgées (environnements résidentiels en forte progression depuis ces dernières années au Québec) sous l'angle de leur marchabilité est proposée. Plus spécifiquement, il s'agit de développer et d'appliquer un audit urbain de manière à proposer un diagnostic morpho-fonctionnel de la marchabilité des environnements résidentiels concernés, puis de rapporter des consultations des résidents pour améliorer la portée explicative de l'outil. Cette première étape d'une recherche plus large a permis de poser les bases d'un croisement entre un diagnostic de la marchabilité, les pratiques de mobilité de résidents et leurs rapports plus sensibles au quartier. Grâce à la construction d'une typologie de marchabilité, l'environnement des complexes résidentiels a été classé selon qu’il soit plus ou moins favorable à la marche, puis ensuite discuté avec des résidents dans le cadre de groupes de discussion. Les résultats ont permis de nuancer l'hypothèse qui proposerait que l'environnement urbain de la ville soit mieux adapté à la marche que ne l'est celui de la banlieue, tout comme l'influence relative des dimensions fonctionnelles sur les perceptions de la marchabilité par les aînés.

Abstract: The aging of populations is suggestive of social changes across several North-American agglomerations, where it is acutely visible in the Post-War suburbs. Among other perspectives, this is a unique opportunity to rethink the urban quality of these living environments, where one depends on the car to get around, and, with a view to a more active mobility. An exploratory analysis of the immediate urban environment for three elderly houses is conducted in order to evaluate their walkability. These types of residential environments have been rapidly developing in recent years. More specifically, we propose an urban audit in order to provide morpho-functional diagnosis of walkability for residential areas, and then to expose discussions conducted with elderly residents to improve the explanatory power of the audit. This first step in a broader research helped to set the basis for crossing the environment walkability diagnosis with the residents' mobility uses and their qualitative relationships to neighborhood. With the construction of a typology of environment walkability, the seniors residential complexes have been classified according to whether a more or less favourable environment for walking, those results discussed with the use of focus groups with the residents. The results allowed us to precise the hypothesis that would suggest the urban environments are better suited to walking than suburban environments, as well as the relative influence of functional dimensions on the perceptions of walkability by seniors. 
Mots clés : Audit urbain - marchabilité - vieillissement - habitat - urbain - banlieue - Montréal

Keywords: Urban audit - walkability - aging - habitat - urban - suburb - Montreal

\section{INTRODUCTION}

Le vieillissement des territoires de banlieue au sein des aires métropolitaines nord-américaines comme Montréal fait apparaître dans un premier temps l'amélioration considérable des conditions socioéconomiques et de santé des personnes vieillissantes et âgées. Les statistiques sur l'espérance de vie, 82 ans pour les femmes et 76 ans pour les hommes, en témoignent à bien des égards (ISQ, 2011). En l'absence de maladies chroniques ou de cancer, cet horizon atteint même près de 86 ans pour les femmes et plus de 80 ans pour les hommes (Martel, Choinière, 2007). Si, en moyenne, on vit plus vieux et en meilleure santé, l'environnement socio-spatial vient selon le contexte multiplier les vieillissements possibles (Charpentier et al., 2010).

Ainsi, plusieurs recherches sur les conditions de vie des personnes âgées ont pu montrer au cours des dernières décennies que les aînés forment un groupe hétérogène (Rosenberg et Everitt, 2001 ; Rosenbloom, 2004) selon, entre autres, le genre, le niveau d'éducation, les catégories socioprofessionnelles ou le revenu. Certains travaux menés sur les espaces métropolitains portant sur l'habitat et les mobilités des aînés, en lien étroit avec la morphologie urbaine et la configuration fonctionnelle des milieux résidentiels, pointent de leur côté plusieurs inégalités spatiales (Cliche et al., 2012; Waldorf, 2003), qui s'ajoutent aux déterminants de la vieillesse déjà bien connus.

Si le vieillissement généralisé dans des milieux urbains variés est évocateur des profonds changements sociaux à l'échelle métropolitaine, il est particulièrement visible au sein des premières couronnes de banlieue. En outre, la présence de cette population vieillissante ${ }^{1}$ dans des territoires étalés, engendre de nouveaux besoins et surtout une nouvelle façon de pratiquer et de penser la ville, tant pour les décideurs que pour la population. Entre autres perspectives, nous considérons qu'il s'agit ici d'une occasion unique de repenser la qualité

1. Population âgée de plus de 60-65 ans mais qui présente des profils socioéconomiques et de santé relativement diversifiés, surtout après 75-80 ans. urbaine de ces milieux de vie, où l'on dépend de la voiture pour se déplacer, dans une perspective de mobilité quotidienne plus active.

\section{LES ENJEUX DES HABITATS ADAPTÉS À LA VIEILLESSE EN BANLIEUE PAVILLONNAIRE}

Les nombreux travaux menés dans divers territoires de banlieue au sein d'agglomérations québécoises (Lord et Després, 2011, 2012; Negron et al., 2012), nord-américaines (Ladd, 2012) ou européennes (Thomann, Pinson, 2002; Rougé, 2005) mettent en exergue la forte dépendance à la voiture de ces territoires et son corolaire : des conditions de marche problématiques. Le vieillissement marqué des premières couronnes de banlieue nord-américaines est maintenant bien documenté, notamment la volonté des aînés d'aujourd'hui, mais également de plus jeunes retraités, de demeurer le plus longtemps possible dans ce type de quartier. Le profond désir d'une vaste majorité de personnes âgées de vieillir dans leur milieu de vie actuel témoigne d'un fort attachement à celui-ci (Després, Lord, 2005), tout comme de leur capacité d'adapter leur mobilité quotidienne (Lord, 2011 ; Negron et Grou, 2012).

Ces habitats « vieillissants » se sont considérablement diversifiés depuis leur édification. Si les formes urbaines de faible (à la limite moyenne) densité demeurent la norme, des formes d'habitat de plus grande densité se sont également développés. D'importants et d'imposants complexes d'habitation (en termes de nombre de logements et de gabarits) surgissent, en misant sur l'adaptation universelle et l'offre de diverses gammes de services à l'intérieur des complexes : de base (ex. services à la personne, aide domestique, urgence santé) et complémentaires (ex. loisirs, restauration, nutrition, sports). D'un niveau de confort variable, ces complexes peuvent être relativement sécurisés en se comparant, à l'échelle de l'îlot urbain, à un quartier résidentiel fermé (gated community) et pouvant contribuer à un processus de ségrégation des aînés (Renaud, 1991 ; 
Rosenbert et Everitt, 2001). Comme le rapportent Clément et al., (2007), ce type de complexes résidentiels est en croissance soutenue dans les premières générations de banlieues pavillonnaires québécoises, mais leur développement s'observe aussi en périphérie urbaine dans plusieurs villes européennes, y compris dans des environnements au climat favorable comme le Sud de la France (Pihet, 2004).

Le vieillissement de la population complexifie la demande en logement. Au Québec, l'offre se spécialise dans des créneaux relativement nouveaux, notamment les appartements mis en marché pour « aînés autonomes », qui leur sont réservés avec certaines conditions d'âge et de santé (Séguin, 2012). Outre les habitations de type appartement en copropriété, des complexes résidentiels pour aînés, de gestion privée ou communautaire/coopérative et partiellement supportés financièrement par l'État, sont aussi mis en marché. Ces marchés au fort développement s'adressent notamment aux personnes âgées désirant quitter un logement de trop grande dimension au profit d'un logis (en location ou en propriété) de dimension plus restreinte et avec possibilité de services adaptés (restauration, activités communautaires, infirmerie, etc.). Cela vient justement répondre à la préoccupation des aînés de conserver plusieurs dimensions de leur « chez-soi » en demeurant dans le quartier où ils ont passé une grande partie de leur vie active : "Ces dernières années, les promoteurs immobiliers du Québec ont privilégié la construction de complexes mieux intégrés dans l'environnement urbain. Afin d'être attractif pour les baby-boomers toute référence au troisième ou quatrième âge a été gommée et les activités proposées se sont diversifiées (installation de cafés, de restaurants, de bars/rencontre, de cinémas... » (Lavigne [2003] dans Clément et al. [2007]).

Il s'agit donc, dans une certaine mesure, d'une densification de la banlieue dictée par l'implantation de copropriétés pour « jeunes retraités » ou pour personnes plus âgées " autonomes" (Gill, 2012). Cela dit, à l'échelle des milieux de vie, les critères de localisation de ces habitats réservés sont laissés à la discrétion des investisseurs. Par ailleurs, l'importante superficie de terrain requise pour développer certains de ces projets résidentiels, constitue une contrainte importante à leur localisation. Ainsi, ces complexes sont souvent implantés dans des milieux relativement enclavés (ex. bordures d'autoroute, boulevards à haut débit de circulation, secteurs résidentiels excentrés, etc.), où l'environnement urbain est souvent peu favorable à la marche.

\section{QUALITÉ URBAINE ET CONDITIONS DE MARCHE POUR LES AÎNÉS}

Sur la base de ces constats, et du contexte particulier de la banlieue, nous proposons une analyse exploratoire de l'environnement urbain immédiat de résidences pour personnes âgées autonomes sous l'angle de leur «marchabilité » (Clifton et al., 2007; Forsyth, Southworth, 2008, Nasar, Evans-Cowley, 2007). Sans s'implanter de manière anarchique, l'inscription spatiale de ces complexes résidentiels suit une logique de promotion immobilière, en s'établissant sur des terrains à faible coût et sans nécessairement prendre en compte des critères de qualité urbaine autre que, dans bien des cas, la seule proximité d'un centre commercial, qui peut alors devenir un atout lors de la commercialisation des logements. Quel est alors le niveau de «marchabilité » de ces habitats et dans quelle mesure les déplacements à pied peuvent constituer une expérience positive pour les aînés qui habitent ces résidences?

Avec l'âge, mais bien davantage avec l'arrivée de limitations d'autonomie, les déplacements à pied ou impliquant la marche sont plus difficiles. Parce que les personnes âgées vont moins loin en marchant, sortent moins de façon imprévue, voire pour certains se déplacent par obligation, l'environnement à proximité du logement prend tout son sens. Comme l'ont déjà souligné Clément et al. dans des travaux menés auprès de personnes âgées de plus de 75 ans vivant en milieu urbain : "Avec l'âge, l'espace urbain devient davantage choisi. Quand on sort, on sait désormais où l'on va : c'est moins la ville globale qui est considérée que des bouts de ville de plus en plus précis » (1998: 232). Les préoccupations, les craintes et les peurs liées aux limitations physiques, sensorielles et cognitives vécues par les aînés peuvent également limiter leurs déplacements. Plutôt que de risquer de tomber ou d'être bousculées, les personnes âgées peuvent choisir de limiter leur mobilité dans le temps et l'espace. La perception positive des espaces de marche est alors primordiale dans la possibilité de marcher ou non. 
Plusieurs facteurs liés à la forme urbaine et à l'aménagement sont susceptibles d'influencer les comportements de marche chez les personnes âgées. D'une part, des aspects subjectifs associés notamment à la perception de l'individu âgé de sa propre santé, ou encore ses représentations du risque, de la sécurité et de sa condition en tant que "personne âgée », sont à prendre en compte (Clément et al., 1996 ; Yerpès, 2002; Banister et Bowling, 2004). D'autre part, des aspects objectifs liés à la condition de santé de l'individu et à sa capacité de mobilité corporelle, comme la vitesse de marche ou le besoin de support (canne, déambulateur, etc.) sont incontournables (Borst et al., 2008; Langlois et al., 1997 ; Wigan, 1995), tout comme la configuration morpho-fonctionnelle de l'environnement urbain pratiqué (Locket et al., 2005). À titre d'exemple, Borst et al. (2008) rapportent sur la base d'études européennes et américaines, comme celles de van Lenthe et al. (2005), de Michael et al. (2006), de Booth et al. (2000) ou de Pikora et al. (2006), que les dimensions de l'environnement bâti (mobilier urbain, pavage, configuration des rues et intersections, temps alloué aux traverses piétonnes, etc.) sont fondamentales dans l'analyse de la marchabilité. Le contexte immédiat des lieux de résidence possède ainsi une influence considérable sur le niveau et l'intensité des activités physiques menées par les personnes âgées (Michael et al., 2006).

Nous explorerons donc l'hypothèse que les environnements " adaptés » à la vieillesse du point de vue de l'échelle du complexe résidentiel peuvent, par une intégration déficiente dans son environnement urbain, offrir une expérience urbaine relativement problématique du point de vue du piéton âgé. Pourtant certaines recherches montrent que la marche constitue une activité physique d'intensité douce susceptible de contribuer au maintien de la santé et à l'expérience positive du vieillissement (Stuck et al., 1999; Banister et Bowling, 2004), ce que plusieurs auteurs désignent de manière normative comme "vieillissement réussi » (Auger, Tedford-Litle, 2002). Le potentiel de «marchabilité » des environnements résidentiels réservés pour les aînés s'avère ainsi incontournable (e.g. Jackson, 2003; Michael et al., 2006; Pikora et al., 2006; van Lenthe et al., 2005).

Nous présentons dans les pages qui suivent une étude exploratoire où les environnements immédiats de trois complexes résidentiels ont été analysés afin de mieux comprendre les conditions de marche offertes dans leur voisinage. Dans une première étape empirique, nous rapportons le travail de développement d'un audit urbain et la réalisation d'un diagnostic morpho-fonctionnel qu'il permet. Dans une deuxième étape interprétative, nous présentons les résultats de groupes de discussion menés avec des résidents des environnements résidentiels explorés de manière à améliorer la portée explicative de l'audit urbain et à ouvrir des pistes de recherche pour les prochaines étapes de nos travaux.

\section{STRATÉgie et MÉTHOde DE RECHERCHE}

Notre étude s'est concentrée sur trois complexes d'habitation réservés aux aînés dans l'agglomération de Montréal, l'un dans une banlieue de première couronne, le Vieux-Longueuil, et développé autour d'un ancien noyau villageois, deux autres dans le quartier La-Petite-Patrie, un ancien quartier ouvrier de la ville-centre et actuellement en processus de «gentrification» (Lavoie et al., 2011). Le choix de deux milieux contrastés du point de vue de leur forme urbaine vise à tester et nuancer l'hypothèse qu'un quartier de la ville-centre serait mieux adapté à la marche qu'un quartier de banlieue pavillonnaire classique, notamment en raison de la meilleure accessibilité aux équipements et services, comme cela a déjà été montré dans diverses recherches (Apparicio et Séguin, 2004; Cliche et al., 2012). Notons ici que nous misons aussi sur ce contraste morpho-fonctionnel pour ajuster et affiner l'audit urbain. Comme cette première partie de notre étude ne vise pas à comparer ni à décrire l'organisation des complexes eux-mêmes, mais bien à développer l'outil de caractérisation de leur environnement immédiat, le mode de gestion des complexes a été mis de côté. Ces complexes ont été sélectionnés en fonction de contacts privilégiés nous permettant un accès collaboratif aux complexes résidentiels dans le but éventuel de réaliser des entretiens et des groupes de discussion.

\section{Description des deux quartiers à l'étude}

Comme le montre la figure 1, les deux milieux résidentiels analysés, témoins de deux périodes d'édifi- 


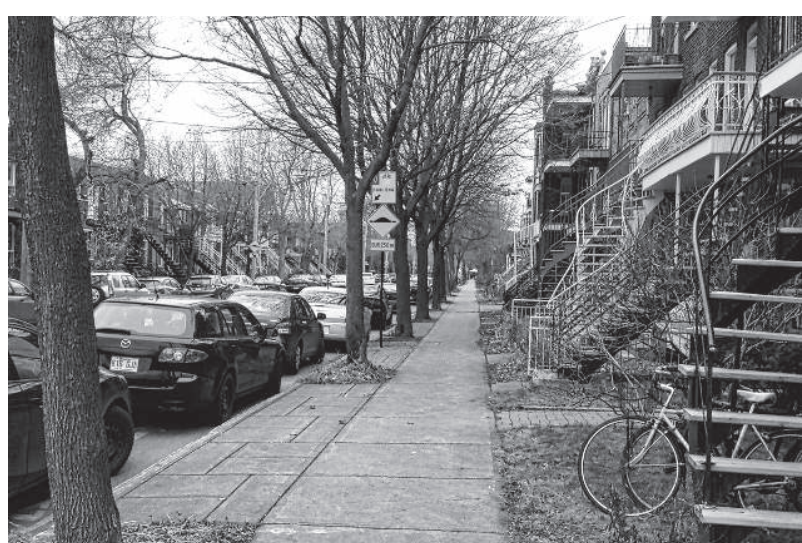

La-Petite-Patrie (cl. S. Lord, 2014)

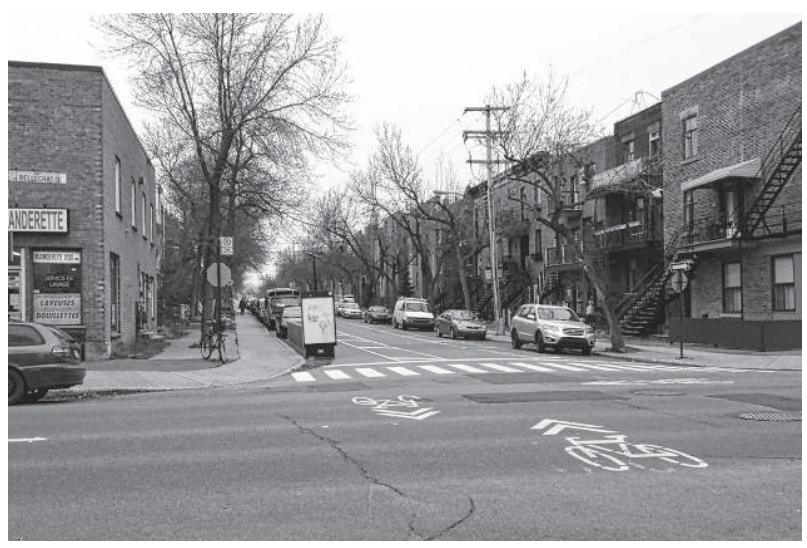

La-Petite-Patrie (cl. S. Lord, 2014)

Figure 1 : Ambiances de rue typiques pour les quartiers analysés Typical street environments for analyzed neighborhoods

cation différentes, présentent des formes urbaines, des organisations fonctionnelles et des ambiances urbaines contrastées. Le quartier La-Petite-Patrie, se caractérise par une forme urbaine offrant plus de variété fonctionnelle où s'entremêlement habitat, activités commerciales et espaces publics, ce qui n'est pas le cas dans le secteur du Vieux-Longueuil où, bien que l'on retrouve des aménités urbaines, les fonctions sont typiquement ségréguées et les distances à parcourir sont significativement plus importantes que pour La-Petite-Patrie.

\section{Les services}

Les avantages qu'offre un quartier plus « urbain » peuvent cependant être nuancés. S’il est vrai que dans le secteur du Vieux-Longueuil les distances sont plus grandes pour rejoindre commerces et services, la présence d'un centre commercial de grand

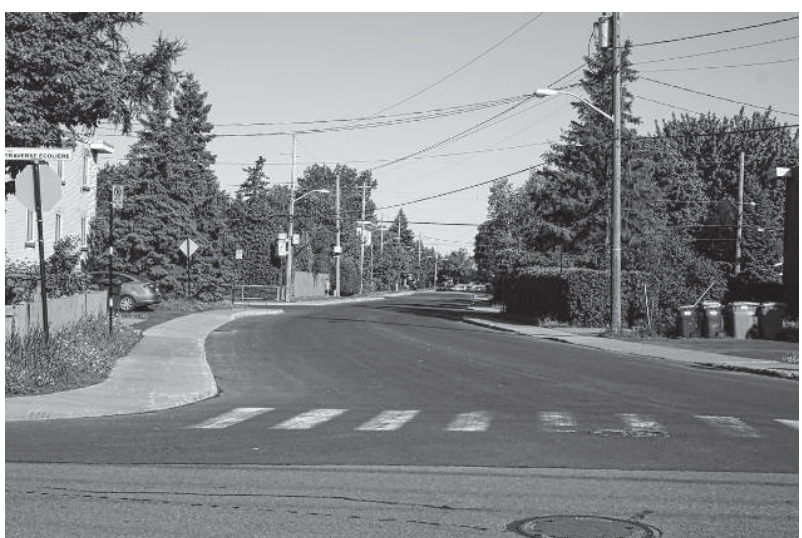

Vieux Longueuil (cl. S. Lord, 2014)

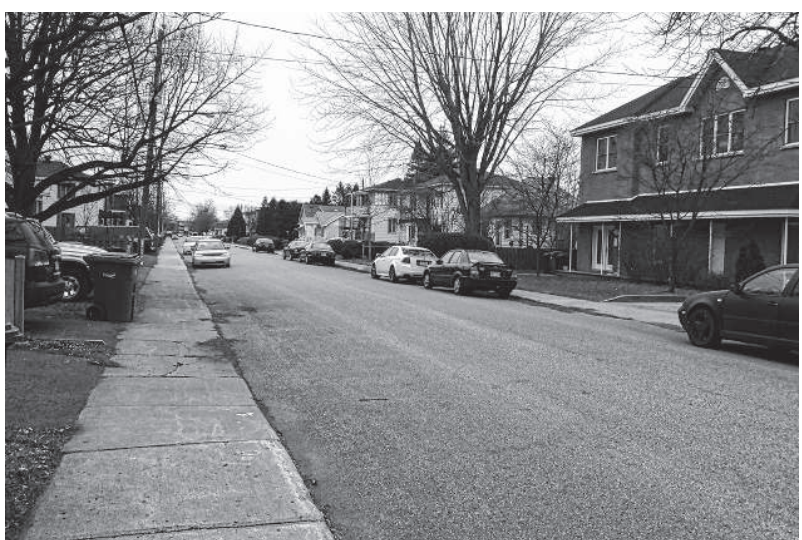

Vieux Longueuil (cl. S. Lord, 2014)

gabarit peut constituer un avantage, puisqu'une fois sur place, tout est localisé au même endroit, ce qui n'est pas le cas dans un quartier de ville-centre comme La-Petite-Patrie. De plus, le centre commercial offre l'avantage d'un environnement couvert, ce qui est tout particulièrement apprécié par les personnes âgées (Lord, 2011), lors par exemple de périodes météorologiques difficiles comme l'hiver ou en temps de canicule l'été.

\section{Les transports}

Dans La-Petite-Patrie, la présence importante de transports en commun (autobus et métro) peut faciliter les déplacements des personnes âgées ne pouvant ou ne désirant pas conduire, ce qui n'est pas nécessairement le cas pour le Vieux-Longueuil. Bien que le transport en commun soit présent, la couverture du territoire est moins importante en 
raison de la faible densité résidentielle et l'offre est organisée principalement en fonction des déplacements en période de pointe. De plus, la présence de bancs et d'abribus est relativement limitée en banlieue alors qu'il s'agit d'un élément plus usuel dans des quartiers centraux comme La-Petite-Patrie, facilitant d'autant les conditions de marche et d'attente des transports en commun des aînés comme pour le reste de la population (Lord, 2004).

\section{Le réseau piétonnier}

La multifonctionnalité du tissu urbain de La-Petite-Patrie et la densité offerte par une trame orthogonale composée d'îlots relativement étroits permet une réduction des distances de marche entre les résidences et les commerces et services environnants, ce qui constitue un avantage pour les déplacements à pied. L'organisation spatiale du secteur entourant le complexe résidentiel du VieuxLongueuil ne se compare pas à la plupart des banlieues typiques, avec des rues curvilignes réduisant la circulation de transit et compliquant d'autant les déplacements piétons. Malgré que la trame de rue soit orthogonale, elle est cependant beaucoup plus relâchée que dans les quartiers centraux, comme c'est le cas de La-Petite-Patrie. Si cela permet de limiter le nombre d'intersections à traverser, source de danger de collision, cela implique également des distances de marche relativement importantes pour rejoindre les commerces et services de proximité (Negron et al., 2012).

\section{La végétation}

Puisque le sentiment de sécurité est un élément important pour les marcheurs âgés, notamment la peur d'accidents, parfois combinée à la peur de chuter, pouvant causer des blessures importantes, l'environnement urbain peut ainsi jouer un double rôle. Dans un premier temps, la présence d'une zone tampon entre le trottoir et la chaussée composée d'arbres ou arbustes, comme par exemple dans le quartier La-Petite-Patrie, peut conférer une certaine sécurité en éloignant le marcheur de la circulation automobile. Cette présence de végétation, ou la présence de bâtiments plus hauts et/ou rapprochés de la voie de circulation permet également de réduire la vitesse automobile. Dans un second temps, la présence d'arbres et de végétation génère une certaine ambiance, une qualité environnementale créée notamment par des zones d'ombre continues. Ce type d'éléments, qu'il s'agisse de zones tampon ou d'arbres, n'est pas présent de manière uniforme dans le secteur à l'étude du Vieux-Longueuil, sauf à quelques endroits ponctuels, particulièrement aux alentours de l'ancien noyau villageois. Le paysage du secteur est plus monotone et les zones d'ombre relativement rares. Cela dit, le calme de la banlieue peut être un avantage face à l'animation urbaine de La-Petite-Patrie, cette dernière pouvant être perçue comme problématique pour les aînés fragilisés en raison, entre autres, des circulations rapides, de la peur de se faire bousculer ou de chuter (Clément et al., 2000).

\section{Les complexes résidentiels pour aînés sélectionnés}

Les résidences pour aînés choisies dans le cadre de cette étude sont représentatives de ce secteur du marché résidentiel. Au Vieux-Longueuil, il s'agit du complexe «Les habitations Paul-Pratt» (figure 2) qui compte 62 appartements individuels, dont 3 de deux chambres et 59 d'une chambre, répartis sur 4 étages. De construction récente, on y retrouve un centre communautaire et un service alimentaire au rez-de-chaussée en communication directe avec une cour privée partagée. Le complexe se veut ainsi un ensemble accessible aux résidents mais aussi aux autres aînés du quartier. Ce centre est géré par un organisme sans but lucratif.

L'organisme sans but lucratif (OSBL) La Maison des Aînés gère trois immeubles de résidences ainsi qu'un centre communautaire pour les personnes âgées du quartier La-Petite-Patrie. L'établissement offre les repas du midi aux résidents ainsi qu'aux personnes âgées du quartier. Sont également offerts des services comme le transport pour un rendez-vous médical ou pour des emplettes au marché public. Le complexe La Maison des Aînés gère 155 logements, répartis dans des immeubles de quatre étages, et accueille environ 175 locataires. Enfin, le Carrefour Hôc-Môn compte un total de 118 unités d'habitation réparties sur six étages. On y retrouve des services de repas sur place dans une salle à manger ainsi que des services de soins. Tout comme les deux autres complexes, le Carrefour Hôc-Môn est 


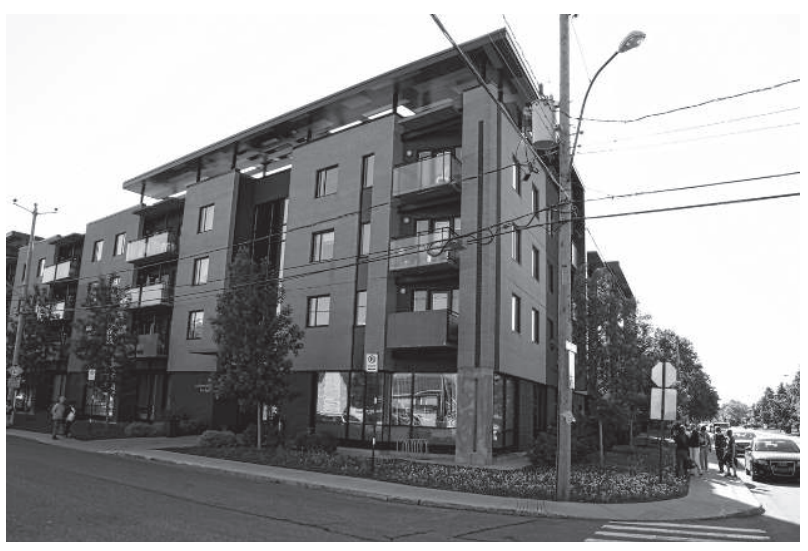

Vieux-Longueuil - les habitations Paul-Prat (cl. S. Lord, 2014)

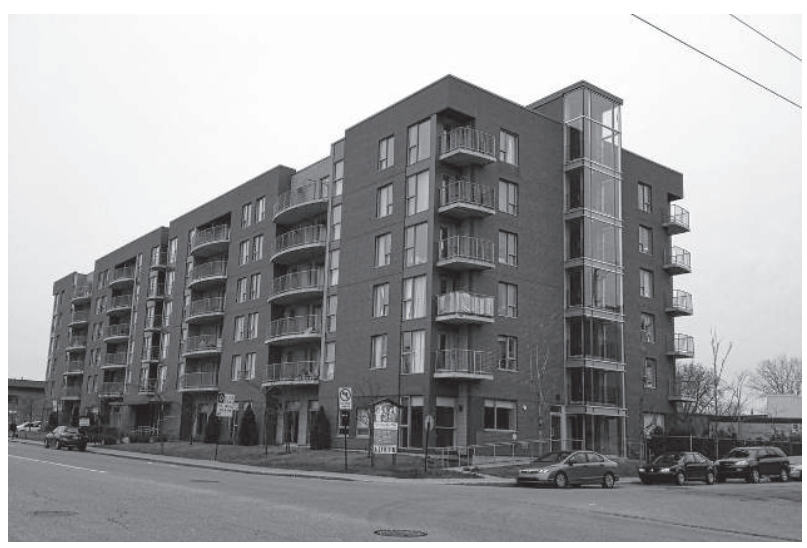

La-Petite-Patrie - le carrefour Hôc-Môn (cl. S. Lord, 2014)

aussi géré par un organisme à but non lucratif. Il accueille à $50 \%$ des résidents de la communauté asiatique. Dans les trois cas, les coûts de location d'un logement ne représentent pas des sommes prohibitives. D'ailleurs, peu importe le prix de location ou d'achat, les résidences pour personnes âgées sont tenues de respecter les mêmes normes gouvernementales en matière de conception architecturale et d'organisation des services.

\section{La délimitation des zones d'application de l'audit}

Pour effectuer l'analyse des environnements urbains autour des trois complexes résidentiels, des zones spécifiques ont été délimitées en utilisant l'application Network Analyst du système d'information géographique $\operatorname{ArcGIS}^{\odot}$ v. 10. À l'aide de cet outil, les zones d'étude ont été délimitées en construisant un «espace de proximité » de $500 \mathrm{~m}$ sur le réseau de rues à partir des centroïdes du

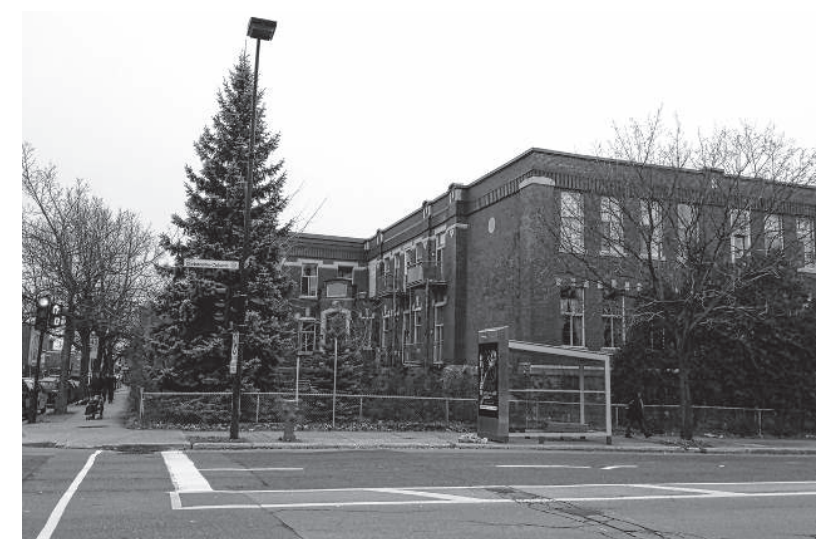

La-Petite-Patrie - la maison des Aînés de la Petite-Patrie (cl. S. Lord, 2014)

Figure 2 : Présentation des trois résidences pour personnes âgées Presentation of the three elderly residential complexes

bâtiment principal des complexes résidentiels. Cette distance est usuellement utilisée (Achuthan, Titheridge, Mackett 2010; Apparicio, Séguin, 2006; Negron et al., 2012) dans l'analyse de l'accessibilité à pied dans un contexte de vieillissement. Ces espaces correspondent à un rayon d'environ cinq ou six minutes de marche autour des complexes d'habitation. Comme le montre la figure 3, cette technique permet de créer des espaces qui prennent la forme d'un polygone irrégulier (et non pas un cercle comme lorsqu'on utilise des distances à vol d'oiseau), permettant ainsi de mieux s'approcher de la réalité des environnements de marche des zones à l'étude.

La caractérisation que nous effectuons sur les espaces de proximité autour des complexes résidentiels sélectionnés prend donc en compte le point de départ des éventuels déplacements des résidents, sans considérer de destinations précises. Nous nous concentrons sur les qualités urbaines d'un environnement spatial potentiel, et non pas sur des mobilités effectives. Cela dit, cette même caractérisation pourrait, entre autres, permette des analyses fines associées à la continuité et à la marchabilité réelle comme des parcours, des cheminements ou des chaînes complètes de déplacements. Cela permettrait en outre de prendre en compte des questions de distances, et d'éventuellement les lier des caractéristiques urbaines et des parcours choisis à des niveaux d'effort ou de capacité pour différents profils de personnes âgées. 


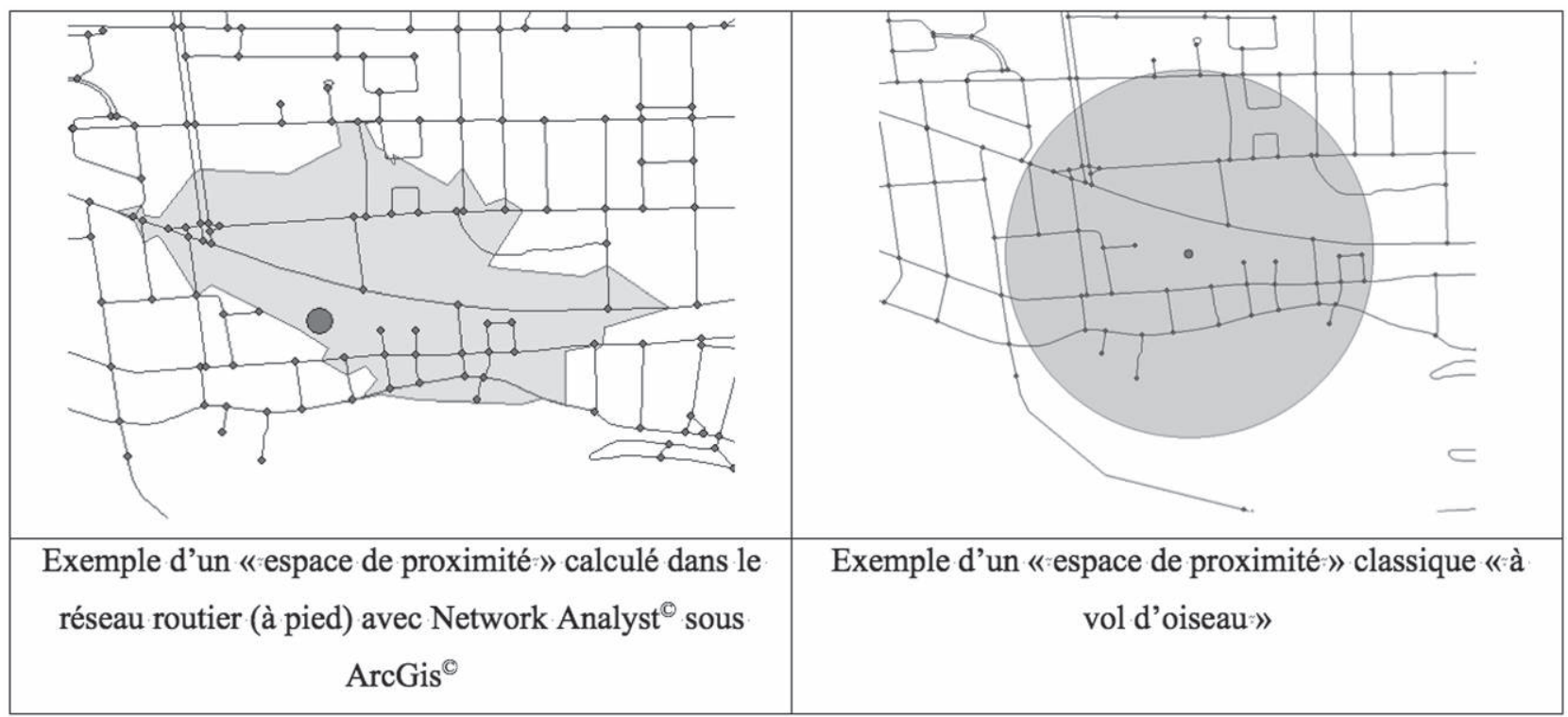

Figure 3 : Méthode de délimitation des zones étudiées (source : Marquet, 2008) Methodology for determining analyzed environments

Une fois les zones d'étude délimitées autour des complexes d'habitation, une seconde étape de préparation a consisté à effectuer la codification de l'ensemble des segments composant la trame viaire. Pour cette opération, nous avons utilisé la base de données du réseau routier de Statistique Canada $(2006)^{2}$ sous format $\operatorname{ArcGIS}^{\odot}$ où un segment correspond à une portion de rue comprise entre deux intersections. Lors des tracés des zones d'étude et de la segmentation de la trame viaire, un certain nombre de segments ont été exclus des analyses. Généralement, ces segments correspondaient aux portions de rues contigües aux zones d'analyse mais exclues des 500 mètres, ou encore à des secteurs où la marche n'est pas possible et/ou autorisée (e.g. bretelles d'autoroute).

\section{La création de l'audit urbain}

L'audit proposé prend appui sur des outils similaires comme le PEDS (Pedestrian Environment Data Scan; voir Clifton et al., 2007). Il a été ajusté pour mieux répondre à la problématique des déplacements à pied des personnes âgées et au contexte de la banlieue dans la région montréalaise. Les ajouts effectués découlent d'une série d'entretiens effec-

2. Disponible à : [http://www12.statcan.gc.ca/census-recensement/2011/geo/ RNF-FRR/index-2011-fra.cfm?year=06]. tués auprès de personnes âgées dans le cadre d'une recherche précédente (Nergron et Grou, 2012; Negron et Lord, 2013). D'autres modifications proviennent d'audits spécifiques aux personnes âgées à savoir le SWEAT (Senior Walking Environmental Assessment Tool; voir Michael et al., 2009) et le NEWS (Neighborhood Environment Walkability Scale; voir Saelens et al., 2003b). L'audit proposé a été structuré en une grille d'observation d'une seule page sur laquelle on retrouve un ensemble de questions et observations, ce qui facilite la saisie de l'information lors des visites de terrain. Quarante-deux (42) questions sont proposées et structurées selon les sept thématiques présentées dans le tableau 1.

Les questions de l'audit visent à documenter de manière détaillée et systématique non seulement les dimensions fonctionnelles et sécuritaires favorables à la marche chez les personnes âgées, mais aussi celles associées à la qualité urbaine. Une attention particulière a été apportée à la simplicité et à l'objectivité des questions, de manière à limiter au mieux l'interprétation des réponses par les différentes personnes effectuant les relevés. En effet, vu le nombre de segments à relever et à observer, et le temps nécessaire pour effectuer les relevés (entre 7 et 9 minutes par segment), le travail de terrain implique plusieurs personnes. Chaque segment a été caractérisé sur chacun des deux côtés de la rue. 


\begin{tabular}{|l|l|}
\hline \multicolumn{1}{|c|}{ Thèmes de l'audit } & \multicolumn{1}{c|}{ Exemple de question/observation } \\
\hline 1. Intersection et aides au piéton (2 par segments) & Présence d'aide au piéton dans l'intersection (Oui/Non/Sans objet)? \\
\hline 2. Caractéristiques de zones tampon & $\begin{array}{l}\text { Caractéristiques dominantes de la zone tampon }(\text { Gazon, Arbre, Ar- } \\
\text { bustes, Pavé, Poteaux, Autres, Sans objet)? }\end{array}$ \\
\hline 3. Caractéristiques des trottoirs & Interruption des trottoirs le long du segment (Oui/Non/Sans objet)? \\
\hline 4. Occupation du sol et caractéristiques des bâtiments & $\begin{array}{l}\text { Présence de commerces avec vitrine dans le segment (Oui/Non/Sans } \\
\text { objet) } \text { ? }\end{array}$ \\
\hline 5. Espace et équipements publics & $\begin{array}{l}\text { Présence d'espace public aménagé dans le segment (Oui/Non/Sans } \\
\text { objet)? }\end{array}$ \\
\hline 6. Caractéristiques de la chaussée & Nombre de voies de circulation et de stationnement sur rue? \\
\hline 7. Entretien et appréciation générale & Présence de déchets dans le segment (Oui/Non)? \\
\hline
\end{tabular}

Tableau 1 : Thématiques abordées dans l'audit urbain Topics addressed in the urban audit

Les relevés de terrain ont été effectués en été et durant la journée uniquement. L'utilisation de questions objectives permet de réduire significativement l'espace d'interprétation, i.e. la variabilité entre les observateurs, et donc d'améliorer la validité de l'outil pour recueillir des caractéristiques urbaines détaillées. D’autres questions de nature plus subjective, faisant intervenir la perception de l'observateur, ont également été intégrées, notamment sur l'appréciation générale des conditions de marche ${ }^{3}$. Notons enfin que les résultats des audits sont intégrés dans un système d'information géographique (SIG) permettant d'effectuer des requêtes et des calculs spécifiques.

\section{LA « MARCHABILITÉ » DES ENVIRONNEMENTS IMMÉDIATS DES COMPLEXES RÉSIDENTIELS}

Pour cette première partie exploratoire, l'application de l'audit dans les territoires du Vieux-Longueuil et de La-Petite-Patrie a permis d'atteindre deux objectifs : 1) caractériser de manière systématique les environnements immédiats des résidences pour personnes âgées ; 2) proposer une typologie de segments de rue plus ou moins favorables à la marche dans un contexte de vieillissement ; 3) confronter nos analyses aux attitudes des usagers concernés, des personnes âgées résidant dans les complexes résidentiels étudiés.

3. Dans l'optique de perfectionner et de valider l'outil, $25 \%$ du total des segments des territoires analysés ont fait l'objet d'un double relevé par des personnes différentes. Les réponses octroyées pour ces segments ont été comparées à l'aide de tests de type Kappa de manière à valider statistiquement la cohérence et la consistance des réponses de l'audit. Les résultats de ces tests sont disponibles dans Negron-Poblete et Lord (2015).

\section{La caractérisation des segments de rue dans les deux environnements urbains}

Dans un premier temps, notre analyse a porté sur certains éléments de l'audit (tableau 2) en lien avec les dimensions objectives favorables à la marche des personnes âgées. Avec ces éléments, des attributs permettant d'évaluer la sécurité et le confort des déplacements à pied ont été considérés. Cette première étape, d'abord descriptive, a permis de poser les bases de la constitution d'une typologie de segments capable de prendre en compte des cheminements, c'est-à-dire de mettre en relation les différents segments des territoires analysés.

Comme le montre le tableau 2, la forme urbaine relâchée de la banlieue ressort de manière saillante pour les Habitations Paul-Pratt dans le VieuxLongueuil, comparativement aux deux autres résidences dans La-Petite-Patrie, un quartier plus urbain. On observe que l'ensemble des segments du complexe résidentiel de banlieue possède une marge avant végétalisée éloignant les bâtiments des zones de marche et en créant une ambiance de marche ouverte, dans une certaine mesure, plus verte qu'en "ville ». En comparaison, seulement 56 \% de segments de la Maison des Aînés et 86 \% des segments du Carrefour Hôc-Môn en quartier central possèdent une telle marge, dont environ un segment sur deux est végétalisé dans le premier cas et près de deux sur trois dans le deuxième.

On compte fort peu de segments, dans les trois cas, où l'on ne retrouve pas de trottoirs, ou bien le fait que ceux-ci soient interrompus. Seul le Carrefour Hôc-Môn se distingue avec, légèrement, moins de trottoirs en mauvais état. Enfin, sur les cri- 


\begin{tabular}{|c|c|c|c|}
\hline Caractéristiques des segments & $\begin{array}{c}\text { Les Habitations Paul-Pratt } \\
(\mathrm{n}=120)\end{array}$ & $\begin{array}{c}\text { La Maison des Aînés de } \\
\text { La-Petite Patrie } \\
(\mathrm{n}=88)\end{array}$ & $\begin{array}{c}\text { Le Carrefour } \\
\text { Hôc-Môn } \\
(\mathrm{n}=106)\end{array}$ \\
\hline Sans trottoir & $1 \%$ & $3 \%$ & $0 \%$ \\
\hline Avec trottoirs discontinu & $5 \%$ & $5 \%$ & $3 \%$ \\
\hline Avec trottoirs en bon état & $73 \%$ & $72 \%$ & $86 \%$ \\
\hline Avec marge avant & $100 \%$ & $56 \%$ & $63 \%$ \\
\hline Avec marge avant végétale & $99 \%$ & $52 \%$ & $42 \%$ \\
\hline Avec zone tampon & $21 \%$ & $88 \%$ & $15 \%$ \\
\hline Avec espace(s) public(s) & $8 \%$ & $57 \%$ & $18 \%$ \\
\hline Avec commerces/services & $28 \%$ & $66 \%$ & $42 \%$ \\
\hline Avec ombrage & $24 \%$ & & 86 \\
\hline
\end{tabular}

Tableau 2 : Attributs évalués dans le cadre de la caractérisation de la marchabilité Assessed attributes for walkability analysis

tères les plus étroitement liés au confort, la Maison des Aînés de La-Petit-Patrie se distingue des deux autres complexes, qu'ils soient en quartier central ou en banlieue. En effet, on retrouve près de $60 \%$ de segments avec une zone tampon entre le trottoir et la chaussée, alors que $42 \%$ de segments du Carrefour Hôc-Môn, et seulement 21 \% de ceux des Habitations Paul-Pratt, présentent une telle configuration. La Maison des aînés se distingue également en ce qui a trait aux segments où l'on retrouve des commerces et des services ainsi qu'au niveau des zones d'ombre. De manière descriptive, le cas de la Maison des aînés, dans un quartier central, semble ainsi offrir une meilleure "marchabilité ", tant au niveau fonctionnel que du confort. Pour leur part, les Habitations Paul-Pratt, en banlieue, semblent offrir un environnement de marche fonctionnellement meilleur que le Carrefour Hôc-Môn, dans un quartier central, mais avec une qualité urbaine moindre que ce dernier.

\section{La création d'une typologie de segments}

Dans un deuxième temps, la série de requêtes descriptives présentées à la section précédente, variable par variable, combine des éléments de l'audit de manière à créer une typologie de segments de rues. En effet, le type d'analyses utilisées habituellement dans les analyses d'accessibilité spatiale (Apparicio et Séguin, 2006; Paez et al., 2010), y compris pour les audits urbains (Clifton et al., 2007 ; Saelens et al., 2003 ), répond plutôt à une perspective fonc- tionnelle et souvent uni-variée. Suite à la description d'éléments factuels, nous avons, dans cette deuxième étape, tenté de dépasser le niveau de la description pour entrer, dans une certaine mesure, dans celui de la compréhension des logiques expérientielles du marcheur âgé. Cette perspective multi-variée était d'autant nécessaire pour répondre à notre troisième objectif, soit d'échanger avec des personnes âgées des territoires étudiés sur des conditions de marche en «milieu naturel ».

Quatre thématiques principales dérivées de la littérature ont été utilisées pour construire la typologie de segments : 1) la sécurité, 2) la fonctionnalité, 3) l'occupation du sol et 4) l'ambiance. Le tableau 3 présente les éléments d'audit ayant été associés et/ ou croisés à chacune d'entre elles.

La typologie de segments proposée est cumulative, un type supérieur possède les caractéristiques des types inférieurs sans toutefois qu'il n'y ait, pour l'instant, de pondération des différents attributs. Le premier type, "Sans confort ni sécurité ", est la configuration la plus défavorable à la marche. Elle présente l'absence de trottoirs sur le segment ou des trottoirs qui sont interrompus. Le deuxième type, "Sécurité minimale », est la configuration exigée au niveau des autorités municipales ${ }^{4}$ et ne présente aucun confort de marche spécifique. Le troisième type, «Confort minimal », est le premier niveau où

\footnotetext{
4. Cette configuration générique varie d'une municipalité québécoise à une autre, mais se limite à la présence d'un trottoir d'au moins 1,2 m (4 pieds) de large sans interruption sur un segment de rue. Cette configuration n'implique aucune aide au piéton aux intersections.
} 


\begin{tabular}{|c|c|}
\hline $\begin{array}{l}\text { Niveau de « marchabilité » } \\
\text { du segment }\end{array}$ & Types de segment \\
\hline \multirow[t]{5}{*}{ Faible } & $\begin{array}{l}\text { Type } 1 \text { - Sans confort ni sécurité. } \\
\text { Segment sans trottoir ou avec un trottoir interrompu. }\end{array}$ \\
\hline & $\begin{array}{l}\text { Type } 2 \text { - Sécurité minimale. } \\
\text { Segment avec trottoir non interrompu. }\end{array}$ \\
\hline & $\begin{array}{l}\text { Type } 3 \text { - Confort minimal. } \\
\text { Segment avec trottoir non interrompu et en bon état et une zone tampon avec la chaussée. }\end{array}$ \\
\hline & $\begin{array}{l}\text { Type } 4 \text { - Confort relatif. } \\
\text { Segment avec trottoir non interrompu et en bon état et une zone tampon végétale avec la chaussée } \\
\text { et une marge avant. }\end{array}$ \\
\hline & $\begin{array}{l}\text { Type } 5 \text { - Tout confort. } \\
\text { Segment avec trottoir non interrompu en bon état, zone tampon avec la chaussée aménagée } \\
\text { (gazon et/ou arbre et/ou arbustes), marge avant végétale ou marge avant non végétale avec espaces } \\
\text { publics et/ou présence de vitrines et présence d'ombrage. }\end{array}$ \\
\hline
\end{tabular}

Tableau 3 : Typologie de segment offrant un plus ou moins grand niveau de «marchabilité » Typology of street segments offering greater or lesser level of walkability

l'on retrouve un élément de confort, soit la présence d'un trottoir continu en bon état en plus de la présence d'une zone tampon éloignant le marcheur de la circulation. Le quatrième type, "Confort relatif », comprend les caractéristiques précédentes, mais vient qualifier la zone tampon en y ajoutant la caractéristique végétalisée et en ajoutant une marge avant éloignant les bâtiments de la zone de marche. Enfin, le cinquième type, "Tout confort ", propose une configuration comprenant l'ensemble des caractéristiques des types précédents en y ajoutant l'aménagement de la zone tampon (gazon et/ou arbre et/ou arbustes), la caractéristique végétalisée de la marge avant en plus d'ombrage et de la présence de commerces ou d'espace public.

La figure 4 montre, qu'une fois croisés en typologie de «marchabilité », les critères semblent confirmer que l'environnement immédiat des Habitations Paul-Pratt est, parmi les trois complexes résidentiels, le plus favorable à la marche. En fait, plus d'un segment sur deux appartient au type « Confort minimal » $(55 \%)$, type qui permet au piéton de marcher dans un environnement avec des trottoirs en bon état et éloignés de la façade des bâtiments. La figure 5 (planche I) montre bien, de manière spatiale, la domination de ce type de segments. Cela dit, près du quart $(23 \%)$ des segments de ce même complexe offre une "sécurité minimale ", et surtout une minorité (13\%) appartient au type «Tout confort». En fait, toujours avec la figure 6 (planche II), on observe que les segments offrant une meilleure «marchabilité » sont localisés face à un centre commercial, segments ayant fait l'objet d'une rénovation récente de la part de la municipalité. Pour rejoindre cette zone, notons que le marcheur peut circuler sur des segments offrant un « Confort minimal » pour rejoindre des commerces et services concentrés en un seul endroit.

Cette qualité de "Confort minimal » du complexe résidentiel de banlieue n'est pas présente pour le Carrefour Hôc-Môn de La-Petite-Patrie, où l'on compte $75 \%$ appartenant au type "Sécurité minimale », cette grande majorité de segments est tout juste positionnée au-dessus des segments «Sans confort ni sécurité ». En fait, même si l'environnement immédiat du Carrefour Hôc-Môn comprend $9 \%$ de segments correspondant au type "Tout confort » et $6 \%$ et à celui « Confort relatif », la figure 6 montre que ces types sont localisés en périphérie du territoire étudié. Le marcheur doit donc circuler sur des segments avec un niveau de «marchabilité » relativement faible pour rejoindre les segments plus confortables et sécuritaires. Cette configuration, dans un espace urbain plus dense, semble apparaître moins favorable à la marche que le cas banlieusard étudié.

Enfin, la Maison des Aînés, toujours dans La-Petite-Patrie, offre l'environnement immédiat le plus favorable en matière de " marchabilité » des trois complexes étudiés. Non seulement compte-telle la plus grande proportion de segments appartenant au type «Tout confort » $(31 \%)$, mais également le plus faible nombre de segments appartenant aux types les plus pauvres en matière de « marchabi- 


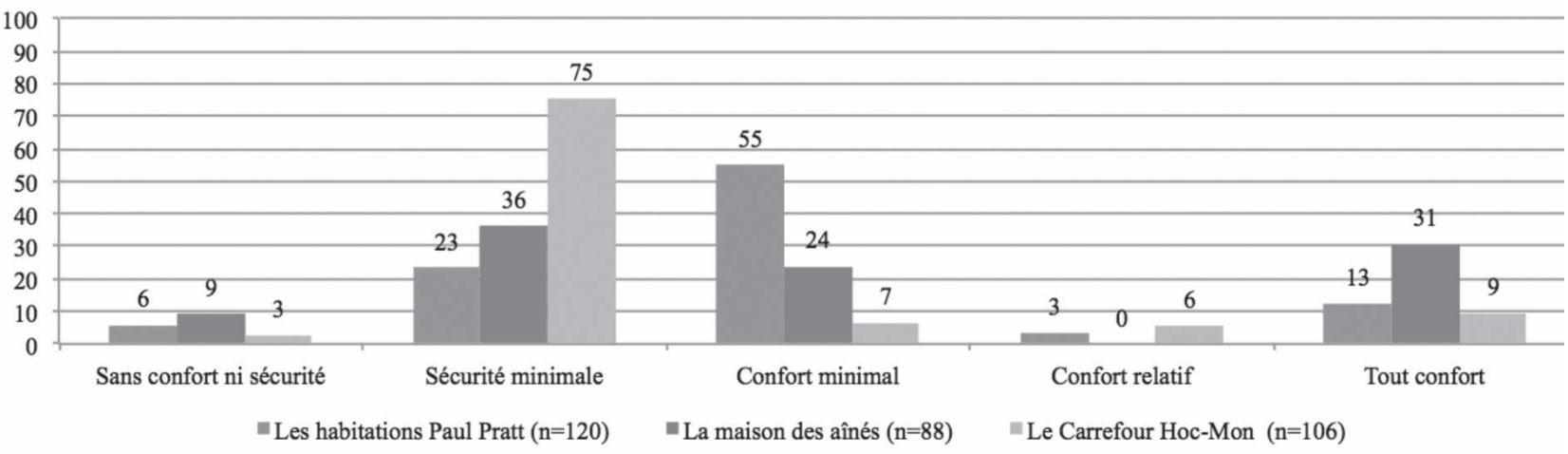

Figure 4 : Typologie de «marchabilité » des environnements immédiats des trois complexes résidentiels (en \%) Typology of walkability of immediate surroundings for the three residential complexes (\%)

lité » $36 \%$ pour « Sécurité minimale » et $24 \%$ pour «Confort minimal»). Qui plus est, la Maison des aînés est localisée au centre de plusieurs segments offrant une bonne condition de marche. Cela dit, on retrouve $9 \%$ de segments appartenant au type le moins favorisé («Sans confort ni sécurité »), segments toutefois concentrés en un seul endroit du territoire étudié. De manière intéressante, contrairement aux Habitations Paul-Pratt, qui compte aussi plusieurs segments du type «Tout confort", la Maison des aînés dans La-Petite-Patrie n'a pas fait l'objet d'une rénovation ciblée. Ces segments où la marche est, selon notre typologie, la plus sécuritaire et confortable pour les aînés, semblent relever d'un aménagement urbain de plus grande qualité que la banlieue, renvoyant en conséquence à des aspects historiques sur les pratiques d'aménagement en lien avec la présence de la voiture mais aussi sur le temps que peut prendre un couvert végétal, le cas échéant, pour en arriver à maturité.

\section{La marchabilité des territoires étudiés selon leurs résidents}

Sur la base des résultats de l'audit et de la typologie proposée, nous avons rencontré des résidents dans chacun des complexes résidentiels étudiés dans le cadre de groupes de discussion (Geoffrion, 2003). Si l'utilisation de cette méthode de recueil de données est discutable quant à l'effet de groupe sur la qualité des données générées, notamment face à l'entretien semi-dirigé, l'un de ses avantages est la rapidité avec laquelle des informations peuvent être recueillies et analysées. Abondamment utilisée dans le cadre de la mise en marché de produit, le groupe de discussion permet d'explorer les attitudes d'un groupe homogène et d'en arriver à certains consensus sur différents objets de discussion, incluant des images. À la résidence Paul-Pratt du VieuxLongueuil nous avons pu rencontrer 4 femmes et 1 homme âgé de 60 à 70 ans. Au Carrefour HôcMôn, dans La-Petit-Patrie, nous avons pu rejoindre 11 femmes et un homme âgés de 76 à 85 ans - tous d'origine vietnamienne, une majorité d'entre eux est arrivée à Montréal à la fin des années 1960-70. Toujours dans La-Petit-Patrie, à la Maison des aînés, nous avons pu discuter avec 14 femmes âgées de 75 à 89 ans.

Dans un premier temps interrogés sur leur mobilité et leurs habitudes de déplacement dans leurs quartiers respectifs, les résidents des trois complexes résidentiels considèrent la marche comme une activité primordiale dans leur quotidien. Structurant de manière fondamentale leur mode de vie, et donc leurs rapports au quartier tout comme leur participation sociale, les moments accordés à la marche visent à « [...] se maintenir en bonne santé, physiquement et moralement ", "pour socialiser, discuter et rencontrer du monde» (commentaires recueillis à la Maison des aînés). À noter, ces déplacements se font tous les jours, parfois plus d'une fois par jour, parce que "c'est bon pour le moral». "[L]a marche a pris encore plus de place depuis qu'on est à la retraite» (commentaires recueillis aux Habitations PaulPratt). Par ailleurs, pour les trois sites investigués, la marche s'effectue le jour uniquement, jamais en soirée, à moins d'y être obligé. 
Les difficultés liées à l'âge et la nécessité, pour certains, d'utiliser des déambulateurs pour se déplacer limitent les distances parcourues. L'accès aux zones de proximité étudiées et le fait de pouvoir s'y déplacer à pied vient, pour plusieurs individus en perte d'autonomie, discriminer les résidants entre ceux ayant la possibilité de maintenir un mode de vie actif et/ou un lien social dans le quartier et les autres qui s'en remettent aux activités offertes à l'intérieur des complexes résidentiels. La qualité urbaine et la diversité fonctionnelle des espaces de proximité que nous avons étudiés prennent ici tout leur sens dans la marchabilité des territoires. Cela s'observe également dans l'utilisation des transports collectifs, du bus ou du métro, assurément davantage dans le quartier La-Petite-Patrie mieux desservi en ce sens que le Vieux-Longueuil. L'usage du transport adapté est un mode de déplacement particulier, en ce sens qu'il vient palier à des problèmes d'accès au territoire, notamment pour des déplacements plus longs comme le centre-ville ou spécialisés comme le médecin, mais n'est pas utilisé au jour le jour.

Lorsque questionnés plus spécifiquement sur les qualités et défauts éventuels des espaces de proximité de leurs résidences, les répondants des deux complexes résidentiels de La-Petite-Patrie ont montré des attitudes significativement différentes de ceux de la banlieue. Le quartier de banlieue du Vieux-Longueuil n'offrait pas selon eux, globalement, un environnement de marche attrayant, non pas pour l'adaptation d'éléments fonctionnels (échelle des rues, trottoirs, mobilier urbain, etc.) comme dans La-Petite-Patrie, mais plutôt pour des questions d'ambiance étroitement associés, d'une part, au manque d'animation et, d'autre part, au manque d'un couvert végétal abondant et mature. La question de la sécurité était relativement prégnante dans leurs commentaires, que ce soit sur le manque d'éclairage, les longues distances à parcourir en cas de problème ou encore les difficultés de cohabitation avec les automobilistes dans des rues avec peu ou pas de signalisation. Les répondants du Vieux-Longueuil concentraient plutôt la marche vers les parcs, les rues commerçantes ou les centres commerciaux, autant d'espaces fréquentés pour la marche de santé et non pas pour des déplacements fonctionnels. Les discussions dans les deux résidences de La-Petite-Patrie ont pour leur part souli- gné l'avantage des courtes distances vers des destinations significatives (parcs et rues commerciales) et la mixité du quartier. La vitesse, le bruit, la rapidité des circulations, tout comme l'exigüité des espaces de marche sont mentionnés à titre d'obstacles à la marche.

Si les attributs constituant la plus ou moins grand marchabilité des segments de la typologie proposée ont semblé pertinents lors des discussions, la qualité de ces espaces de marche doit selon les résidents reposer sur des critères beaucoup plus complexes. La question des ambiances ressort de manière saillante, notamment sur l'attractivité de certaines destinations. La simple présence de trottoirs ne rend pas nécessairement plus «marchable» un segment donné : "J'ai les chevilles fragiles, puis si le trottoir n'est pas droit, ça me fait peur, il faut toujours être attentif à où on met les pieds » (commentaires recueillis aux Habitations Paul-Pratt). La question de l'entretien des espaces de marche (trottoirs, intersections, etc.), rend possible ou non les marches quotidiennes - et dans le cas de la saison hivernale elle peut contraindre l'ensemble des déplacements à l'intérieur du complexe résidentiel pendant plusieurs jours. Ainsi, les marches sont plus fréquentes l'été que l'hiver. Néanmoins, les contraintes posées par la neige et la glace sont moins soulevées en centre-ville qu'en banlieue, sans doute du fait du contexte urbain plus central et compact dans lequel s'insèrent les complexes résidentiels. Mentionnons enfin que les conditions de pratique de la marche mentionnées par les résidents comportent des différences spécifiques qui, malgré des similarités, sont propres au contexte urbain de chaque espace de proximité étudié. En ce sens, des intersections plus ou moins faciles à traverser, la présence de pistes cyclables offrant un espace de marche plat et sécuritaire en heure creuse ou bien une terrasse ou un café viennent considérablement moduler la fréquence et le caractère agréable de la pratique de la marche.

\section{Conclusion et prochaines ÉTAPES DE RECHERCHE}

Grâce à la construction de notre typologie de « marchabilité » en utilisant notre audit urbain, nous pouvons affiner notre hypothèse de départ qui proposait que l'environnement de la ville soit mieux 
adapté à la marche que ne l'est celui de la banlieue. En effet, les types de rues les plus confortables ne sont pas nécessairement objectivement plus présents dans le quartier central La-Petite-Patrie que dans celui du Vieux-Longueuil, en banlieue. Contrairement à ce que nous envisagions, même si le secteur urbain semble plus confortable, la configuration de la banlieue n'est finalement pas complètement différente. Qui plus est, des opérations récentes de rénovation visant l'amélioration des conditions de marche ont été relevées par l'outil proposé. Nos groupes de discussion vont dans le même sens que ces constats, en limitant peut-être la portée des constats issus de notre typologie. Si l'on marche peut-être autant en banlieue qu'en centre-ville, on ne marche pas pour les mêmes raisons et pas non plus vers les mêmes destinations. Point d'observation évidemment attendu, ce constat développé par nos discussions avec les résidents des complexes résidentiels étudiés nous suggère d'ajouter d'autres attributs à notre typologie. Il serait aussi possible de la compléter par d'autres couches d'informations issues de l'audit impliquant des zones de territoire plus ou moins favorables à la marche, voire plus ou moins favorable en matière d'attractivité.

Néanmoins, la qualité urbaine intrinsèque du quartier de ville-centre semble dominer, même sans réfections majeures des rues. Cette perspective de comparaison morpho-fonctionnelle banlieue-centre sera à explorer dans la conduite d'audits supplémentaires. Cela dit, nous sommes d'avis que des enquêtes, notamment qualitatives pouvant inclure des techniques de type parcours commentés (i.e. Chaudet, 2012), auprès des usagers sur des parcours empruntés ou bien des cheminements potentiels, sont une perspective particulièrement porteuse. En effet, de pouvoir croiser l'expérience du marcheur avec des données objectives et détaillées sur son environnement permettrait d'approfondir la notion même de marchabilité, tout comme certains facteurs explicatifs des choix de cheminements dans la ville. Dans une perspective plus appliquée de l'aménagement et de l'urbanisme, c'est bien ce type de connaissances qu'il apparaît opportun de générer pour adapter les environnements urbains.

Nos travaux montrent qu'une analyse plus fine s'avère aussi pertinente, à l'intérieur même de chacun des territoires étudiés - et ce à une échelle encore plus près des marcheurs. De fortes dispa- rités semblent exister au sein d'un même territoire d'étude, peut-être même plus qu'entre les territoires différents, angle que nous n'avions pas jusqu'ici privilégié mais que les groupes de discussion ont mis en lumière. En effet, les configurations urbaines offertes par certains types de segments tirent vers le haut ou bien vers le bas le niveau de " marchabilité » de l'ensemble d'un territoire étudié, et ce qu'il s'agisse d'un quartier de la ville-centre ou de la banlieue. Par exemple dans le Vieux-Longueuil, des sections du boulevard chemin-de-Chambly, près du centre commercial Place Jacques-Cartier, ont subi des interventions en vue de les rendre plus agréables à la marche (création d'une zone tampon végétale, élargissement des trottoirs). Ceci rend ces segments, à l'image de plusieurs rues de La-Petite-Patrie bien, pourvus en aménités urbaines et de mobilier urbain, clairement positifs au niveau de la «marchabilité », mais moins significatifs au niveau des préférences environnementales au niveau des usagers. Encore ici, le croisement de données d'enquête avec l'audit urbain apparaît opportun pour donner davantage de force compréhensive au constat objectif de la marchabilité de la typologie. Une documentation plus précise et détaillée de configurations contextualisées doit être réalisée non pas seulement en ville-centre mais également en banlieue.

L'audit que nous avons construit au cours de cette première étape de recherche, encore bien exploratoire, permet d'identifier de manière systématique des éléments morphologiques et fonctionnels de l'environnement urbain qui peuvent être en faveur, ou encore aller à l'encontre, de la marche pour les personnes âgées. En effet, les diagnostics que nous avons effectués par l'intermédiaire de notre grille d'analyse permettent d'explorer une liste d'éléments qu'il semble pertinent de prendre en compte pour rendre les environnements urbains plus adaptés, plus sécuritaires et plus confortables à la marche des aînés. À ce titre, notre recension des écrits doit être affinée et croisée à des analyses plus approfondies de territoires supplémentaires.

Plus fondamentalement, cette analyse exploratoire montre que de nombreuses choses sont à faire en banlieue, mais aussi en centre-ville, pour adapter la «ville » au vieillissement démographique du Québec. Les aînés développeront des nouveaux rapports à l'environnement, notamment en matière de mobilité et d'accessibilité, forçant les décideurs 
de la ville à renouveler leurs façons de penser et de faire la ville. En même temps, cette analyse fine et détaillée, et qui sera prolongée sur d'autres territoires, soulève l'hypothèse qu'une intervention lourde et globale, et donc plus coûteuse, sur l'ensemble des environnements de banlieues n'est peut-être pas nécessaire ni forcément efficiente pour répondre au vieillissement de la population. En outre, notre approche ouvre la voie à une meilleure compréhension de l'environnement bâti pour mieux cibler, par exemple, des secteurs spécifiques à densifier ou à mieux équiper en matière de mobilier ou d'équipement urbain.

Enfin, il convient de rappeler qu'une telle exploration fine et détaillée, avec l'application d'un audit urbain, qu'elle soit étendue à plus ou moins de territoires, demeure essentiellement descriptive. Elle permet, au minimum, de rendre compte des caractéristiques morpho-fonctionnelles d'un espace, et au mieux d'informer la prise de décision relative à l'entretien ou à l'application de rénovation normée. Cela dit, ce même audit urbain offre un potentiel de compréhension considérable s'il est croisé avec la consultation de résidents ou de décideurs. C'est bien l'analyse des pratiques et des représentations de l'espace bâti qu'il semble ici pertinent de mieux comprendre pour créer des milieux où la marche est facile, sécuritaire et confortable. C'est ce que nous ferons dans la phase suivante de cette recherche. Après avoir exploré ces trois environnements immédiats de complexes résidentiels pour aînés, nous prolongerons l'application de cet audit sur d'autres habitats du même type mais également sur des parcours empruntés par des personnes âgées ainsi que certaines destinations clés.

\section{Remerciements}

Les auteurs tiennent à remercier Brice Marquet et Amélie Renard pour leur contribution au projet en tant que stagiaires à l'Observatoire de la mobilité durable à l'été 2012, ainsi que Geneviève Grou, candidate à la maîtrise en urbanisme à l'Institut d'urbanisme de l'Université de Montréal. Le réseau Ville, Région, Monde (VRM) et l'Observatoire de la mobilité durable de l'Institut d'urbanisme sont également remerciés pour leur appui financier au projet.

\section{Bibliographie}

Achuthan K., Titheridge H., Mackett R. L., 2010. Mapping accessibility differences for the whole journey and for socially excluded groups of people, Journal of Maps 6 (1), p. 220-229.

Apparicio P., SÉguin A., 2006. L'accessibilité aux services et aux équipements : Un enjeu d'équité pour les personnes âgées résidant en HLM à Montréal, Cahiers de géographie du Québec, 50, 139, p. 23-44.

ArcGis (v. 10), Système d'information géographique, New York, ESRI.

Auger J.A., Tedford-Litle D., 2002. From the Inside Looking Out, Halifax, Fernwood publishing.

Banister, D., Bowling A., 2004. Quality of life for the elderly: the transport dimension, Transport Policy, 11, 2, p. 105-115.

Booth M.L., Owen N., Bauman A., Clavisi O., Leslie E., 2000. Social-cognitive and perceived environment influences associated with physical activity in older Australiens, Preventive Medicine 31, p. 15-22.

Borst H.C., Miedemaa H.M.E., de Vriesb S.I., Grahama J.M.A., van Dongena J.E.F., 2008. Relationships between street characteristics and perceived attractiveness for walking reported by elderly people, Journal of Environmental Psychology 28, p. 353-361.

Chaudet B., 2012. Les territoires du « bien vieillir » au prisme de la mobilité quotidienne des personnes âgées, in VIRIOTDurandal J.-P., Pihet C., Chapon P.-M. (dir.), Les défis territoriaux face au vieillissement, Paris, La documentation française, p. 17-30.

Clément S., Mantovani J., Membrado M., 1998. L'urbain dans tous ses états : Faire, vivre et dire la ville, in Hautmont N. (dir.), Habitat et sociétés, Paris, L'Harmattan, p. 231-254.

Cliche L., Séguin A.-M., Apparicio P., 2012. Qualité de l'environnement urbain autour des résidences privées pour personnes âgées à Montréal : un portrait multidimensionnel, in Negron-Poblete P., SÉguin A.-M. (dir.), Vieillissement et enjeux d'aménagement : regards à différentes échelles, Presses de l'Université du Québec, Montréal, Québec, p. 67-98.

Clifton K. J., Smith A. D. L., Rodriguez D., 2007. The development and testing of an audit for the pedestrian environment Landscape and Urban Planning, 80, p. 95-110.

Després C., Lord S., 2005. The meaning of home for elderly suburbanites, in Rowles G.D., Chaudhury H. (dir.), Home and Identity in Late Life: International Perspectives, New York, Springer, p. 317-340.

Forsyth A., Southworth M., 2008. Cities Afoot - Pedestrians, Walkability and Urban Design, Journal of Urban Design, 13(1), p. 1-3.

Geoffrion P., 2003. Le groupe de discussion, in Gauthier B. (dir.), Recherche sociale. De la problématique à la collecte de données, Québec, Presses de l'Université du Québec, $4^{\mathrm{e}}$ édition p. 333-356.

Gill D., 2012. Ville et vieillissement, in Beaudet G., Meloche J.-P., Scherrer F., Questions d'urbanisme, Montréal, Presses de l'Université de Montréal.

ISQ (Institut de la statistique du Québec), 2011. Le bilan démographique du Québec, Édition 2011, Québec, Institut de la statistique du Québec. 
JACKSON L.E., 2003. The relationship of urban design to human health and condition, Landscape and Urban Planning 64, p. 191-200.

LAdD A., 2012. Stranded in suburbia: Planning for aging populations, Canadian Center for Policy Alternatives, Manitoba Office, (consulté le 26 avril 2013, [http://www.policyalternatives.ca/sites/default/files/uploads/publications/Manitoba\%20 Office/2012/02/Suburbia\%20final.pdf]).

Langlois J.A., Keyl P.M., Guralnik J.M., Foley D.J., Marottoli R.A., Wallace R.B., 1997. Characteristics of older pedestrians who have difficulty crossing the street, American Journal of Public Health, 87, p. 393-397.

Lavoie J. P., Rose D., Burns V., Covanti V., 2011 . La gentrification de La Petite-Patrie. Quelle place et quel pouvoir pour les aînés ?, Diversité urbaine, 11(1), p. 59-80.

Lord S., Després C., 2011 . Vieillir en banlieue nordaméricaine : le rapport à la ville des personnes âgées, Gérontologie et Société (136), p. 189-204.

Lord S., Després C., 2012. Vieillir dans la ville étalée : enjeux, éléments de solution et défis de mise en œuvre, in NEGronPoblete P., SÉguin A.-M. (dir.), Vieillissement et enjeux d'aménagement: regards à différentes échelles, Montréal, Presses de l’Université du Québec, p. 119-154.

Lord S., 2011 . Le choix de vieillir à domicile : L'inévitable adaptation des modes de vie, Retraite et société (60), p. 199-213.

LORD S., 2004. Les aînés face à leur avenir résidentiel : le cas de résidents de banlienes de Québec, Québec, Mémoire de maîtrise, Université Laval.

Martel S., Choinière M., 2007. Une estimation du fardeau de différentes maladies chroniques à partir de l'espérance de vie ajustée en fonction de l'état de santé, Québec, Institut national de santé publique.

Marquet B., 2012. Étude de l'environnement urbain autour des résidences pour personnes âgées autonomes. Paris, École des ingénieurs de la Ville de Paris, Rapport individuel de stage d'étude et recherche [Montréal].

Michael Y. L., Green M. K., Farquhar S., 2006. Neighbourhood design and active aging, Health $\&$ Place, 12, p. 734-740.

Michael Y. L., Keast E. M., Chaudhury H., Day K., Mahmood A., SARTE A. F. I., 2009. Revising the senior walking environnemental assessment tool, Preventive Medecine, 48, p. 247-249.

Nasar J., Evans-Cowley J., 2007. Universal design and visitability, from accessibility to zoning, Columbus, The John Glenn School of Public Affairs.

Negron P., SÉguin A.-M., Apparicio P., 2012. L'accessibilité à pied pour les résidents âgés du Vieux-Longueuil, des problèmes à l'horizon, in Negron-Poblete P., Séguin A.-M. (dir.), Vieillissement et enjeux d'aménagement : regards à différentes échelles, Montréal, Presses de l'Université du Québec, p. 155-178.

Negron, P., Lord S., 2013. La mobilité des aînés dans le périurbain à Montréal : tirer profit de l'environnement urbain pour promouvoir un vieillissement plus actif, Communication présentée dans le cadre des Deuxièmes rencontres internationales du Forum des Vies Mobile « Des mobilités durables dans le périurbain, est-ce possible? », La Maison rouge, Paris, 24 et 25 janvier 2013.
Negron-Poblete P., Grou G., 2012. Mobilité chez les aînés habitant les banlienes de première couronne : projets de mobilité chez les aînés de Lachine et du Vieux-Longueuil Communication présentée dans le cadre du Colloque « Formes de mobilités et inclusions : quelles relations? ", $80^{\text {e }}$ congrès de l'ACFAS, 7-8 mai 2012.

Negron-Poblete P., Lord S., 2015 (à paraître). Marchabilité des environnements urbains autour des résidences pour personnes âgées de la région de Montréal : Application de l'audit MAPPA, Les Cahiers de géographie du Québec.

Paez A., Mercado R. G., Farber S., Morency C., Roorda M., 2010. Accessibility to health care facilities in Montreal Island: an application of relative accessibility indicators from the perspective of senior and non-senior residents, International Journal of Health Geographics, 9, 1, 15 p.

Pinet C., 2004. Les populations âgées en France au début du $\mathrm{XxI}^{\mathrm{e}}$ siècle : dynamiques démographiques et questions sociales, Vie et Vieillissement, 3(2), p. 48-54.

Pikora T.J., Gilles-Corti B., Knuiman M.W., Bull F.C., Jamrozik K., Donovan R.J., 2006. Neighbourhood environmental factors correlated with walking near home: Using SPACES, Medicine E Science in Sports E Exercise, 38, p. 708-714.

Rosenberg M., Everitt J., 2001. Planning for aging populations: inside or outside the walls, Progress in Planning, 56, p. 119-168.

Rosenbloom S., 2004. Mobility of the Elderly: Good news and bad news, Transportation research board, Transportation in an Aging Soceity, United States, p. 3-21.

Rougé L., 2005. Inégale mobilité et urbanité par défaut des périurbains modestes toulousains, EspacesTemps.net (consulté le 26 avril 2013, [http://www.espacestemps.net/ articles/inegale-mobilite-et-urbanite-par-defaut-des-periurbains-modestes-toulousains]).

Saelens B.E., Sallis J.F., Frank L.D., 2003, Environmental correlates of walking and cycling: Findings from the transportation, urban design, and planning literatures, Annals of Behavioral Medicine, 25, p. 80-91.

Saelens, B. E., Sallis J. F., Black J. B., Chen D., 2003 b. Neighborhood-based differences in physical activity: an environment scale evaluation, American Journal of Public Health, 93, 9, p. 1552-1558.

Stuck A.E., Walthert J.M., Nikolaus T., Bula C.J., НohMANN C., BeCK J.C., 1999, Risk factors for functional status decline in community-living elderly people: A systematic literature review, Social Science \& Medicine, 48, p. 445-469.

Pinson D., Thomann S., 2002, La maison en ses territoires. De la villa à la ville diffuse, Paris, L'Harmattan, coll. «Villes et Entreprises ».

van Lenthe F.J., Brug J., Mackenbach J.P., 2005. Neighbourhood inequalities in physical inactivity: The role of neighbourhood attractiveness, proximity to local facilities and safety in the Netherlands, Social Science E Medicine 60, p. 763-775.

Waldorf B., 2003, Automobile Reliance Among the Elderly: Race and Spatial Context Effects, Growth and Change, 34, 2, p. 175-201.

Wigan M., 1995, Treatment of walking as a mode of transportation, Transportation Research 1487, p. 7-13. 